for the first immunisation Freund's complete adjuvant was used while for the second to fifth immunisation we use Freund's incomplete adjuvant, in the sixth week only the antigen was used. At week 0,3 and 6, rabbits were bled for the evaluation of the immune response by the indirect ELISA. The final bleeding was performed and the serum obtained was stored at $-20^{\circ} \mathrm{C}$ until use. For he purification of the antibodies we use a protein $\mathrm{A}$ purification kit and the integrity of the immunoglobulins was verified by electrophoresis, the titration of the obtained polyclonal antibody was performed by indirect ELISA. We evaluated the antibody by Western blot, Dot blot and inhibition of hemagglutination using as antigen the ATCC 14018 of G. vaginalis.

Results The first evaluation of the immune response of rabbits showed that prior to immunisation the rabbits had no antibodies against VLY, whereas during the third and sixth week of immunisation they had antibody titres of 1:250 and 1:1000 respectively. Electrophoresis of the polyclonal antibody showed the purity and integrity of the purified antibodies, this antibodies can be use at titers of 1:1000 for the subsequent assays. The western blot showed that this antibody recognised a band of approximately $56 \mathrm{kDa}$ that matched with the molecular weight reported for the VLY, whereas the Dot blot showed that the antibody recognises the VLY of ATCC 14018 of $G$. vaginalis. To corroborate that the antibody inhibited erythrocyte lysis, hemagglutination inhibition assays were performed and we showed that the use of this antibody decreased the cell lysis in around $80 \%$.

Conclusion We produce a polyclonal antibody agains the VLY of G. vaginalis capable of inhibiting the erythrocyte lysis. This antibody will be useful in investigating the role of VLY in the pathogenesis of $G$. vaginalis during the development of BV.

\section{P1.29 ATTACHMENT OF THE SYPHILIS SPIROCHETE, TREPONEMA PALLIDUM, TO THE VASCULAR ENDOTHELIUM}

${ }^{1}$ Karen V Lithgow, ${ }^{2}$ Wei-Chen Kao, ${ }^{1}$ Rebecca Hof, ${ }^{2}$ Helena Petrosova, ${ }^{3}$ Yi-Pin Lin, ${ }^{1}$ Martin J Boulanger, ${ }^{3}$ John Leong, ${ }^{1} J$ ohn E Burke, ${ }^{2}$ Tara J Moriarty, ${ }^{1}$ Caroline E Cameron. ${ }^{1}$ University of Victoria, Victoria, Canada; ${ }^{2}$ University of Toronto, Toronto, Canada; ${ }^{3}$ Tufts University School of Medicine, Boston, USA

\subsection{6/sextrans-2017-053264.137}

Introduction: Treponema pallidum is the causative agent of venereal syphilis, a human-specific sexually transmitted infection characterised by multi-stage disease and diverse clinical manifestations. T. pallidum undergoes rapid hematogenous dissemination, accessing distant organ sites and penetrating tissue, placental, and blood-brain barriers. Tp0751 is an adhesin that interacts with the host vasculature and mediates bacterial adherence to endothelial cells under shear flow conditions. This study explores Tp0751-mediated adhesion to the vascular endothelium.

Methods Tp0751, expressed in a non-infectious model spirochete [Borrelia burgdorferi (Bb-Tp0751)], was assessed for a gain-of-function adhesion phenotype using attachment assays. Interaction specificity was probed with competitive inhibition studies using synthetic peptides of Tp0751 host-binding regions. Affinity chromatography coupled with mass spectrometry was used to identify endothelial receptors for Tp0751. Membrane receptors isolated from human umbilical vein endothelial cells (HUVECs) were incubated with Tp0751-affinity columns and interacting proteins were identified with mass spectrometry.

Results Here we demonstrate that $B b$-Tp0751 adheres to HUVECs under stationary conditions. The laminin receptor (LamR) was identified as an endothelial receptor for Tp0751. LamR is a brain endothelial receptor for other neurotropic invasive pathogens, including Neisseria meningitidis. Current investigations will validate the Tp0751-LamR interaction and characterise the functional outcomes of Tp0751 adhesion to endothelial cells.

Conclusion These investigations reveal the mechanics of T. pallium attachment to endothelial cells, the fundamental step in the process of $T$. pallidum vascular dissemination. A complete understanding of this process will provide opportunities to prevent $T$. pallidum attachment to the host vasculature to facilitate syphilis vaccine development.

\section{P1.30 IN VITRO ACTIVITY OF GEPOTIDACIN AND OTHER ANTIMICROBIALS AGAINST MYCOPLASMAS AND UREAPLASMAS}

Ken Waites, Donna Crabb, Lynn Duffy Li Xiao. University of Alabama At Birmingham, Birmingham, AL, USA

\subsection{6/sextrans-2017-053264.138}

Introduction Mycoplasma and Ureaplasma spp. are important pathogens of the respiratory and urogenital tracts. Antimicrobial resistance limits treatment options. Gepotidacin (GEP), a novel triazaacenaphthylene topoisomerase II inhibitor that inhibits DNA replication by a mechanism and target distinct from fluoroquinolones was tested against 85 isolates of $\mathrm{MycO}$ plasma pneumoniae (Mp), Mycoplasma hominis (Mh), Mycoplasma genitalium (Mg), Ureaplasma parvum (Up), and Ureaplasma urealyticum (Uu) in comparison to azithromycin (AZI), clindamycin (CLI), tetracycline (TET), levofloxacin (LEV), and moxifloxacin (MOX). Organisms tested included strains known to be resistant to TET, LEV, and/or AZI. This work was supported by GSK and funded through OTA HHSO100201300011C with HHS/BARDA.

Methods MICs were determined using broth microdilution in accordance with Clinical and Laboratory Standards Institute Guidelines.

Results GEP was active against $25 \mathrm{Mp}$, MIC range 0.032$0.125 \mu \mathrm{g} / \mathrm{ml}$, including 5 that were AZI-resistant, with $\mathrm{MIC}_{90}$ $(0.125 \mu \mathrm{g} / \mathrm{ml})$, equivalent to MOX. GEP was active against $10 \mathrm{Mg}$. $\mathrm{MIC}_{90}(0.032 \mu \mathrm{g} / \mathrm{ml})$ was 4 -fold < MOX. GEP MICs against $25 \mathrm{Mh}$ ranged from 0.5 to $2 \mu \mathrm{g} / \mathrm{ml}$ with $\mathrm{MIC}_{90}=2 \mu \mathrm{g} /$ $\mathrm{ml}$, making it less active than other agents, including MOX $\left(\mathrm{MIC}_{90}=0.125 \mu \mathrm{g} / \mathrm{ml}\right)$, with exceptions of $1 \mathrm{LEV}$ and 2 TETresistant organisms, for which GEP MICs were unaffected. GEP was less active against 25 Ureaplasma spp. (MIC range $1-8 \mu \mathrm{g} / \mathrm{ml}), \quad \mathrm{MIC}_{90}=8 \mu \mathrm{g} / \mathrm{ml}$. There was no effect on GEP MICs in 9 Ureaplasma spp. with resistance to LEV, AZI, and/ or TET. GEP minimum bactericidal concentrations for 4 isolates of $\mathrm{Mg}, 4 \mathrm{Mh}, 4 \mathrm{Mp}, 3 \mathrm{Uu}$, and $1 \mathrm{Up}$ were $>3$ dilutions $>$ MICs, indicating bacteriostatic effect.

Conclusion GEP warrants further study to treat infections due to Mycoplasma spp., particularly organisms resistant to other antimicrobials as it was active against isolates resistant to AZI, TET, LEV, and/or MOX. 